\title{
INFANTILE VOMITING AND DIARRHOEA
}

\author{
By G. H. Newns, M.D., M.R.C.P. \\ Physician, Hospital for Sick Children, Great Ormond Street
}

A classification of the chief causes of diarrhoea and vomiting in infancy is given below. Diagnosis of the cause of vomiting in infancy can only be arrived at after a careful consideration of the signs and symptoms.

\section{Vomiting}

\section{Obstructive}

Congenital hypertrophic pyloric stenosis.

Congenital atresia or stenosis of the intestine.

Oesophageal stenosis.

Achalasia of cardia of stomach.

Intussusception.

\section{Non-Obstructive}

Overfeeding.

Due to aerophagy.

Rumination.

Habit vomiting.

Allergic vomiting.

Vomiting secondary to infection (often associated with diarrhoea).

\section{Vomiting and Diarrhoea}

I. Gastroenteritis. Infection of the bowel with pathogenic organisms.

II. Due to dyspepsia.

III. Secondary to parenteral infection.

\section{Obstructive Vomiting}

\section{Congenital hypertrophic pyloric stenosis}

This is the commonest of all the congenital forms of obstruction of the gastrointestinal tract.

Although the hypertrophy of the muscle of the pyloric canal is present at birth, symptoms do not start immediately but appear after an: interval of from Io days onwards, and may sometimes be delayed for as long as six-eight weeks. There is no completely satisfactory explanation of the delay in the appearance of symptoms. Pyloric spasm is an important factor in the causation of the obstruction and this does not apparently occur immediately after birth. Symptoms disappear at about the end of the fourth month though the hypertrophy persists for many months longer.

Congenital pyloric stenosis has a much greater incidence in males than femalesabout ten to one-and it is much commoner in first-born babies. It occasionally occurs in more than one child in a family and it has been reported in both of identical twins.

The onset of vomiting is generally abrupt and the mother may be able to name the actual day of onset. Previous to this time the baby has gained weight and thrived. The vomiting rapidly assumes a projectile character, the vomit sometimes being thrown several feet. Fluid is often regurgitated through the nose. The child usually vomits after each feed, though not all the vomits are projectile. The vomit is copious, contains undigested food, but no bile.

The infant is usuaily constipated because very little food is passing into the intestine. In some cases frequent small green stools are passed consisting mostly of bile and intestinal dèbris (so-called hunger stools).

Loss of weight may be considerable and varies with the severity of the obstruction. In the more severe cases the infant becomes very dehydrated. 、

Although this triad of symptoms, projectile vomiting, loss of weight and constipation, is almost pathognomonic of pyloric stenosis, confirmation of the diagnosis is essential. This may be done by observing visible peristalsis. 
and feeling the pyloric tumour. The infant should be examined during a feed. Waves of gastric peristalsis can be seen passing obliquely from left to right. The pyloric tumour can be felt in all cases but considerable experience is required to detect it. It is only palpable when the pylorus is in spasm, and it may be necessary to watch the baby during several feeds before it can be felt. It feels like a small. knob, of the consistency of cartilage, usually situated just beyond the edge of the $R$. rectus but sometimes high up below the edge of the liver. The tumour disappears as the spasm passes off.

In doubtful cases, where a tumour cannot definitely be felt, a barium swallow should be given. This will demonstrate distension of the stomach with marked delay in the emptying time.

\section{(i) Surgical Treatment}

This is the method of choice in most cases. It results in a rapid cure, and the operative mortality in skilled hands is low. It can be performed under a local anaesthetic. In severe cases with much loss of weight and dehydration operation is imperative. Before operation, dehydration must be treated by means of subcutaneous or intravenous saline infusions and the stomach should be washed out once or twice with normal saline (not with sodium bicarbonate, which may precipitate alkalosis).

There are many different règimes for feeding after operation but the principle of all is the same, viz., administration of dilute milk feeds in small amounts frequently at first, gradually increasing in strength and amount. Normal feeds should be reached two days after operation.

\section{(ii) Medical}

This is not new since it was perforce the only treatment (short of gastro-enterostomy) before the introduction of Rammstedt's operation. It was re-introduced on the Continent partly because operation was so often refused. Medical treatment should be confined to the cases where operation is refused, where skilled surgery is not available, and in the milder cases, which generally have a late onset and do not lose much weight.
The main lines of modical treatment are :-

I. Administration of small frequent feeds.

2. Daily gastric lavage with normal saline to reduce the gastritis which is always present.

3. Administration of an anti spasmodic to abolish pyloric spasm. Atropine was employed until recently but this often caused toxic symptoms. Eumydrine (atropine methyl nitrate), which is much less toxic, is used nowadays. It is given either in a $I$ in 10,000 solution, I. 5 to 5 cc., or as lamellae (pylostropine) in $1 / 45^{\circ}$ or $\mathrm{I} / 75^{\circ} \mathrm{gm}$. doses. The lamellae are placed under the tongue. Both preparations are given 20 minutes before each feed.

Medical treatment should be continued until the age of four months, when the symptoms spontaneously disappear.

\section{Obstruction due to other congenital anomalies}

Congenital intestinal stenosis or atresia may cause severe vomiting in the neonatal period and should always be borne in mind when severe vomiting starts soon after birth. The site of the obstruction may be in the duodenum or in any part of the intestine. The lower down the obstruction is, the later the onset of vomiting.

In duodenal stenosis or atresia the vomiting commences very soon after birth, is persistent and often projectile. The vomit, unlike that of congenital pyloric stenosis, contains bile. Visible peristalsis may be present. It is distinguished from pyloric stenosis by the earlier onset and the presence of bile in the vomit, and the absence of a pyloric tumour. A barium swallow will show the ballooned duodenum. Occasionally these infants have survived a gastro-enterostomy.

Similar symptoms are met with in intestinal atresia, which often extends over a considerable length of gut. The actual site is frequently determined only at autopsy.

\section{Oesophageal Atresia}

This is a rare congenital anomaly which gives rise to regurgitation when the first feeds are given. Choking and cyanosis are frequently associated symptoms. The oesophagus usually ends blindly above, the lower 
end being connected to the trachea by a fistula. In one variety the upper end communicates with the trachea. In such cases the infant rapidly develops a fatal aspiration pneumonia.

Recent advances in thoracic surgery have resulted in cures following end-to-end anastomosis.

\section{Achalasia of cardia of stomach}

This is a rare form of obstruction in infancy but possibly commoner than is generally thought. Four cases have come under my observation in the past year. The condition is probably the same as that met with in older children and adults, and is due to a failure of relaxation of the cardiac sphincter.

The baby regurgitates food forcibly soon after it is swallowed and loses weight rapidly. Diagnosis is made by barium swallow. The oesophagus is dilated and the barium is seen to be held up at the lower end of the oesophagus.

Treatment consists in dilatation of the oesophagus two or three times a day with a mercury bougie. In the cases observed, gradual improvement has occurred.

\section{Intussusception}

In the common form of intussusception, vomiting is not as a rule a prominent symptom in the early stages, though later on vomiting may be severe, and faeculent in type. In the rarer enteric type vomiting occurs much earlier.

The infant typically has severe attacks of colicky pain with pallor and sweating. There is usually bright red blood in the stools. The sausage-shaped intussusception can generally be easily felt.

Occasionally in infancy intestinal obstruction may be due to congenital bands, volvulus, or strangulated inguinal herniae.

\section{Non-obstructive Vomiting}

Many babies regurgitate a small part of the feed, especially if the feed is rather large-it is a means of getting rid of an excess. Some infants are very prone to regurgitate, and the volume brought up may be considerable. They generally gain weight nevertheless. Improvement usually results when cereal feeding is introduced. In bad cases the feeds should be considerably thickened with a food such as Benger's or Savory and Moore's.

\section{Overfeeding}

Over distension of the stomach by too frequent feeding or too large volumes may give rise to vomiting in certain babies. It must not be assumed that babies who habitually vomit are necessarily overfed. Underfeeding with associated aerophagy is a much commoner cause of vomiting.

\section{Vomiting from excessive air swallowing}

This is the commonest cause of vomiting in early infancy. The vomiting may be occasional or very frequent; the amounts brought up may be small or large. Characteristically the baby vomits small quantities at intervals between the feeds.

Some babies are inveterate wind swallowers even when adequately fed. They are restless, irritable, hungry infants as a rule. The vomiting is usually associated with attacks of colic which make the baby still more restless. The vomit is never truly projectile though at times it may be explosive, since it is often brought about by a violent eructation of wind. If the baby is underfed constipation may be present or there may be green ' hunger' stools. Loss of weight may also occur for the same reason.

There may be a superficial resemblance to congenital pyloric stenosis but examination of a baby during a feed will reveal neither visible peristalsis nor a tumour.

It must first be determined whether the infant is underfed by a calculation of the correct amount based on the infant's expected weight* ( $2 \frac{1}{2}$ ozs. per lb. of body weight per day or 50 calories per pound). If breast-fed,

\footnotetext{
* The expected weight may be calculated by subtracting 10 from the age of the infant in days, and adding this figure to the birth weight. This holds good up to about the age of four months, after which the gain in weight is less than an ounce a day.
} 
the infant must be test-weighed for $24-48$ hours.

An underfed baby will be so hungry that he will gulp down a large quantity of air during the first few minutes of feeding.

If the baby is underfed the amount of food must be increased to the required level, calculations of the amount being made on the expected, not the actual weight of the baby. In the case of breast-fed infants, complementary feeds must be given, each feed being a test feed. The hole in the teat should be large enough to enable the infant to take the feed easily without straining, in IO-I 5 minutes. A boat-shaped bottle is often better for this type of infant.

A sedative before feeds is very helpful. It makes the baby less restless so that the feed is taken more quietly. Chloral, $\frac{1}{2}-\mathrm{I}$ gr. may be given 20 minutes before each feed. Care should be taken to get up as much wind as possible after feeds.

\section{Rumination. Merycism}

Though not a common form of vomiting it may be very persistent and troublesome. It occurs in breast- and bottle-fed infants alike. The rumination has to be watched for carefully as the baby will not usually perform the act when the mother or nurse is present.

About an hour after feeds the infant is observed to bring up part of the feed, some being swallowed again, some spat out, while some of the food is held in the mouth for some time. The regurgitation is preceded by chewing movements of the jaws. The weight lost is not as a rule very great, generally it remains stationary.

Treatment is difficult. Thickening of the feeds with cereals so that it is of a porridge-like consistency is sometimes successful. A restraining bandage or cap with tapes tied under the chin, and kept on for I-2 hours after feeds may prevent regurgitation. Sedatives may be of value.

\section{Allergic vomiting.}

Occasionally vomiting in infancy is due to allergy to cow's milk, very small amounts of which may give rise to severe vomiting. In the milder cases gradual introduction of milk to the diet will usually desensitize the infant. In the transition period the baby may be given such foods as Benger's, cereals and vegetables, etc., made up with water instead of milk.

In the more severe cases it may be necessary to give milks containing no milk protein. The basis of these milks is usually soya bean flour, with added fats, sugar and minerals.*

Vomiting in infancy may be due to meningitis or cerebral tumour or to toxic states such as uraemia secondary to severe congenital renal disease.

\section{Vomiting associated with Diarrhoea}

Diarrhoea with vomiting is due to three main causes.

\section{(I) Dietetic}

Incorrect feeding or persistent overfeeding, excess of fat or carbohydrates will give rise to diarrhoea and vomiting. Excess of protein causes vomiting, especially due to excessive curd formation. Today, with the increase in knowledge of infant dietetics, severe diarrhoea and vomiting from this cause is uncommon. If neglected however, dehydration will occur and the infant may become as gravely ill as infants in the next group.

\section{(2) Infection of bowel with pathógenic organisms (epidemic gastro-enteritis)}

This was the cause of the one-time summer diarrhoea so prevalent in the hot summers in this country. In this form it is rarely seen nowadays. Epidemics of gastro-enteritis still occur in new-born babies in nurseries. Many cases of diarrhoea arising in hospital wards are of this type and are due to cross-infection. Sonne dysentery is also prevalent in young children and may occur in infants. In hospital the disease may spread rapidly.

\section{(3) Symptomatic diarrhoea and vomiting due to parenteral infection}

Infection outside the bowel is a common cause of diarrhoea and vomiting in infancy.

* There is, at present, no such food made in this country. 
The mechanism is not properly understood. Pyelitis, otitis media, infections of the throat, to name only a few, will give rise to diarrhoea and vomiting, which may in fact be the presenting symptoms. The primary cause may only be discovered after careful search.

The symptoms vary greatly with the severity of the infection. The second group comprises the worst cases. In these rapid dehydration and prostration occur. The stools are watery with very little faecal material, and vomiting is persistent, even water not being retained. The mortality is high. Severe fatty changes are found in the liver at autopsy. Jaundice may occur before death.

In most of these cases no pathogenic organism can be isolated from the stools.

Treatment in the milder cases consists in withdrawing food for 12-24 hours, giving only glucose and saline in small amounts at frequent intervals. There is often a marked improvement within a few hours; the diarrhoea diminishes and the vomiting stops. Milk feeds should then be gradually introduced starting with a weak mixture and gradually increasing the strength. It is not as a rule necessary to give special feeds.

Where dehydration is present more vigorous treatment is required. These cases should, in fact, be regarded as a medical emergency. Parenteral fluid is essential, the only satisfactory route being the intravenous one. All fluids should be stopped by mouth and intravenous glucose-saline, or Hartmann's solution given by drip. This may be continued if necessary for several days. Sodium chloride must only be given in sufficient amounts to replace salt loss, otherwise oedema will occur.

After the vomiting and diarrhoea has stopped, weak feeds should be given cautiously, and gradually increased in strength. Lactic acid milks are especially useful and are well tolerated.

\section{Drug therapy}

Little success has been obtained with sulphonamides and penicillin in straightforward cases of gastro-enteritis, though sulphaguanidine appears to be of value in Sonne dysentery. Appropriate chemotherapy is, of course, indicated when there is a parenteral infection.

Other drugs are of little value. Purging with castor oil has been given up. Astringents such as bismuth and kaolin have little effect. Opium is a dangerous drug to give to dehydrated infants and is seldom used nowadays. 